Boletín de la Sociedad Geológica Mexicana

VOLUMEN 61, NÚM. 2, 2009, P. 225-232

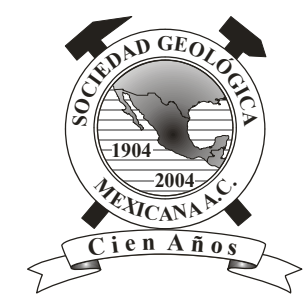

\title{
Tortugas fósiles del Pleistoceno tardío de Santiago Chazumba, Oaxaca
}

\author{
J. Alberto Cruz ${ }^{1, *}$, Joaquín Arroyo-Cabrales ${ }^{1}$ y Ramón Viñas-Vallverdú ${ }^{2}$ \\ ${ }^{1}$ Laboratorio de Arqueozoología “M. en C. Ticul Álvarez Solórzano”, Subdirección de Laboratorios y Apoyo Académico del Instituto \\ Nacional de Antropología e Historia. Moneda 16, Col. Centro, Mexico D. F. 06060. \\ ${ }^{2}$ Institut Català de Paleoecologia Humana i Evolució Social (IPHES), Tarragona, España. \\ *solounrayitodesol@hotmail.com
}

\section{Resumen}

La fauna paleoherpetológica del Pleistoceno de México se compone de 37 géneros y 58 especies registradas para 27 localidades diferentes; en el caso de los reptiles las tortugas son el grupo más abundante en cuanto a registros se refiere. En este trabajo se reportan y describen los restos óseos de Gopherus y Kinosternon aff. K. hirtipes/K. integrum procedentes del municipio de Santiago Chazumba, Oaxaca. El hallazgo de estas especies para el Pleistoceno de Oaxaca permite proponer un ambiente de matorral xerófilo como el existente actualmente en el Valle de Tehuacán-Cuicatlán, pero con una precipitación menor y una temperatura mayor, condiciones similares a las predominantes en el norte del país..

Palabras clave: Distribución, Gopherus, Kinosternon, Pleistoceno, Oaxaca.

\begin{abstract}
The Paleoherpetological Pleistocene fauna record for Mexico is composed by 37 genera and 58 species recorded for 27 different localities; in the case of the reptiles, turtles are the most abundant group. In this paper, we report and describe the bones of Gopherus

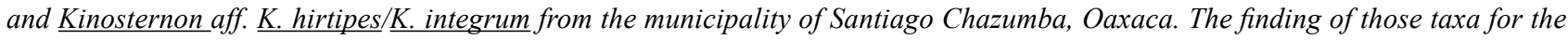
Oaxacan Pleistocene suggest xerophilous scrub environment such the one found today in the Tehuacán-Cuicatlán Valley, but with lower rainfall and higher temperatures, conditions similar to those prevailing in northern México.
\end{abstract}

Keywords: Distribution, Gopherus, Kinosternon, Pleistocene, Oaxaca. 


\section{Introducción}

México es uno de los países megadiversos del planeta $\mathrm{y}$, entre los grupos mejor representados están los anfibios y los reptiles (aquí referidos como herpetofauna), que con 973 especies actualmente conocidas (Liner y Casas-Andreu, 2008) lo colocan como el país con mayor diversidad en el mundo. Para el pasado los registros sobre herpetofauna proceden de 27 localidades con 37 géneros y 58 especies de herpetofauna para el Cuaternario del país (Tovar y Montellano, 2006). La ausencia de registros de herpetofauna fósil se puede explicar por la falta de interés en los estudios paleontológicos sobre los anfibios y reptiles, incluyendo las pocas revisiones realizadas sobre su osteología en nuestro país (Cruz Silva, 2009).

El interés sobre los fósiles de los anfibios y reptiles en México se ha incrementado desde finales del siglo XX, aunque la mayoría de los trabajos han sido publicados como tesis, resúmenes de congresos y guías de campo (Reynoso, 2006). En el caso de los reptiles fósiles en México, las tortugas son los organismos más abundantemente representados en el registro y, dentro de este grupo el género Kinosternon presenta la mayor cantidad de hallazgos, con 18 registros publicados, seguido por Gopherus con 12; sin embargo, ninguno de estos registros procede del Estado de Oaxaca (Reynoso, 2006). En este trabajo se reportan y describen los restos óseos de tortugas procedentes de las excavaciones realizadas en el municipio de Santiago Chazumba, Oaxaca.

\section{2. Área de estudio}

El yacimiento de donde procede el material estudiado se localiza al noreste del municipio de Santiago Chazumba, en el estado de Oaxaca, en una de las barrancas que forma la Cañada o Barranca del Muerto. El sitio se encuentra en las proximidades del pueblo, a un lado de donde están iniciando la construcción de nuevos asentamientos, ubicándose a los $18^{\circ} 11^{\prime} 44^{\prime}$ ' latitud N y 1740'15" longitud O, a una altitud de $1774 \mathrm{~m}$, en un ambiente semidesértico con matorral xerófito (Figura 1), en los límites de la Reserva de la Biósfera del Valle de Tehuacán-Cuicatlán. El sitio paleontológico contiene varios restos óseos en taxa extintos $(\dagger)$ o existentes de vertebrados, incluyendo un †proboscídeo (Proboscidea), $\dagger$ Eremotherium sp. (perezoso terrestre gigante), $\dagger$ Equus sp. (caballo pleistocénico), Microtus sp. (metorito), Neotoma sp. (rata de campo) y las tortugas, todo lo cual se recuperó en un área de excavación de 4 x 6 metros y de 5.3 a 7 metros de profundidad desde la superficie hasta el nivel VII. Este último nivel está compuesto por dos capas: a) arcilla dura y compactada con gran concentración de cantos de caliza, espesor irregular en torno a $80-90 \mathrm{~cm}, \mathrm{~b}$ ) limo arcilloso con arena, grava, cuarzo y, con menor cantidad de rocas que el superior, su consistencia es menos compactada que el anterior (Figura 2). Todavía se desconoce la profundidad
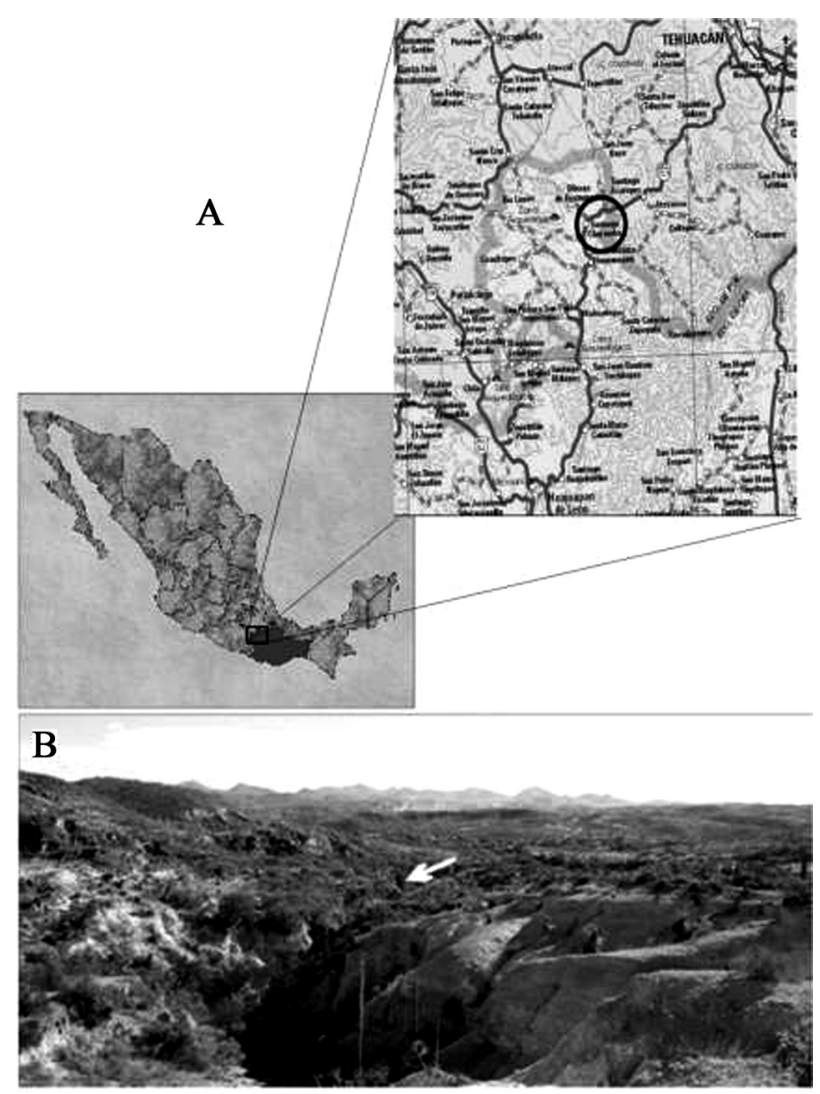

Figura 1. A, Ubicación del sitio; B, Barranca del Muerto. La flecha indica el lugar donde se encontraron los restos fósiles

total del nivel VII.

El material fue depositado en el Laboratorio de Arqueozoología "M. en C. Ticul Álvarez Solórzano", Instituto Nacional de Antropología e Historia (INAH), en la ciudad de México. Los restos fueron recuperados in situ o al tamizar las muestras de sedimento. Los materiales están referidos a la localidad de Chazumba (CH-No. del material); las medidas fueron tomadas con un vernier digital Fowler Ultra-Cal II; el material fue comparado con material osteológico reciente del laboratorio antes mencionado, acrónimo DP y, el arreglo sistemático de los taxa se realizó siguiendo a Flores-Villela (1993) y Flores Villela y CansecoMárquez (2004).

\section{Acrónimos}

Material osteológico de referencia, del Laboratorio de Arqueozoología “M. en C. Ticul Álvarez Solórzano” (DP), utilizado en este trabajo para la identificación de los restos fósiles. DP 6662 Rhinoclemmys cf. areolata, DP 6551 Pseudemys (= Trachemys) scripta, DP 7251 Kinosternon flavescens, DP 5285 Kinosternon hirtipes, DP, 6811, 5169 Kinosternon integrum, DP 5035 Kinosternon scorpioides cruentatum, DP 1595 Gopherus sp. 


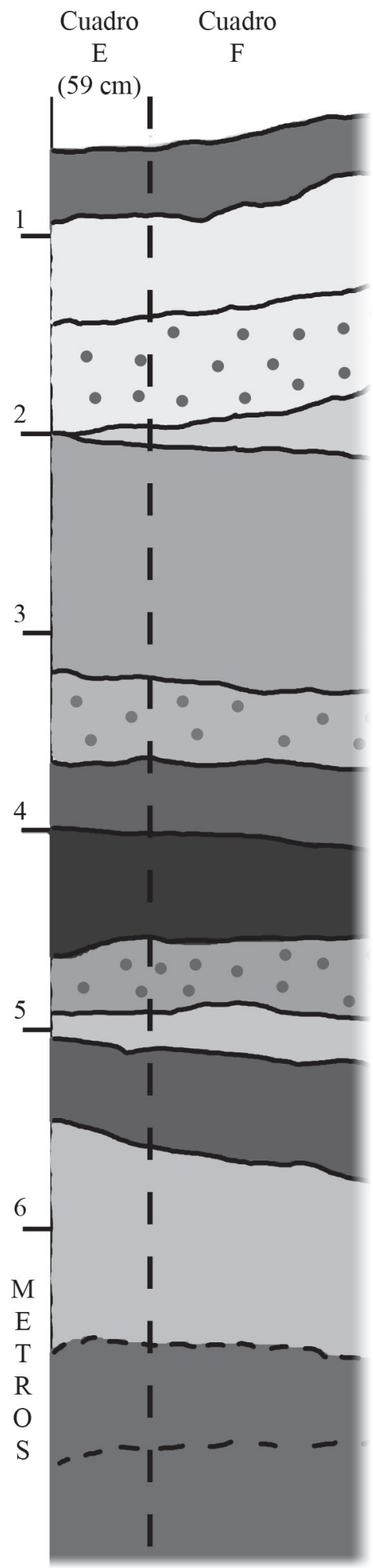

Nivel superior superficial

Arcilla rica en materia orgánica. Espesor $30 \mathrm{~cm}$.

Nivel I a

Limo. Espesor $50 \mathrm{~cm}$. (Blancuzco).

\section{Nivel I b}

Limo y arenas con pequeños cantos rodados. Espesor $50 \mathrm{~cm}$ (Blancuzco)

Nivel II

Limo con arenas y pequeños cantos rodados. Espesor irregular entre $0-50 \mathrm{~cm}$. (Rosado).

Nivel III a

Limo con arenas y pequeños cantos rodados. Espesor entre 30-40 cm. (Grisáceo)

Nivel III b

Limo con arenas y pequeños cantos rodados. Espesor $30 \mathrm{~cm}$. (Gris claro).

Nivel IV a

Arcillas muy compactas. Espesor entre 50-60 cm. (Rojizo)

Nivel IV b

Limo y arcilla compacta. Espesor 50-60 cm. (Marrón rojizo)

Nivel IV c

Limo y arcilla con pequeños cantos rodados. Espesor $30 \mathrm{~cm}$. (Grisáceo).

Nivel V

Limo de espesor irregular. (Beige).

Nivel VI a

Arcilla compactada. Espesor $30 \mathrm{~cm}$. (Marrón).

\section{Nivel VI b}

Limo y arenas. Espesor $100 \mathrm{~cm}$. (Rosado).

Nivel VII a (nivel paleontológico)

Arcilla compactada con gran concentración de caliza. En este nivel se localizaron restos de roedores, una costilla de un posible camélido y pequeños fragmentos no identificados. (gris verdoso).

Nivel VII $b$ (nivel paleontológico)

Limo arcilloso con arena, grava, cuarzo y abundantes piedras. Su consistencia es menos compactada que la anterior. En este nivel se localizaron los restos del mamut, así como tortuga y caballo. Espesor por determinar.

Figura 2. Estratigrafía del sitio. La línea punteada separa los cuadros de los cuales fue tomada la estratigrafía. 


\section{Sistemática Paleontológica}

Reptilia Laurenti, 1768

Testudines Batsch, 1788

Testudinidae Gray, 1825

Gopherus Rafinesque, 1832

\section{Gopherus sp.}

Material recuperado. Un hioplastron izquierdo, $\mathrm{CH}$ 14; una placa costal, $\mathrm{CH} 8$; una primera placa costal derecha, $\mathrm{CH} 11$; un xifiplastron derecho, $\mathrm{CH} 2$ (Figura 3).

Descripción. El fragmento del hioplastron izquierdo tiene un ancho máximo de 56.6mm y una longitud máxima de $68.5 \mathrm{~mm}$. Presenta una forma general cuadrangular, ventralmente existe un escote axilar en forma de $\mathrm{V}$, con un ángulo de $45^{\circ}$, extendiéndose posteriormente un tercio del hioplastron, presentando la parte basal de la proyección axilar que se une al caparazón. Aunque tenuemente, se pueden apreciar las impresiones de los escudos húmeropectoral y pecto-abdominal; el primero se encuentra en la parte anterior de la placa de manera inclinada, originándose en el margen medial e inclinándose anteriormente hacia el escote axilar; la impresión pecto-abdominal se encuentra en la parte posterior de la placa en forma horizontal. La distancia entre las impresiones es más corta en la parte medial y va aumentando conforme se dirige a la parte lateral de la placa (Figura 3A).

La primera placa costal derecha $(\mathrm{CH} 11)$ esta representada por la parte basal, no presenta la impresión del escudo pleural; de la otra costal $(\mathrm{CH} 8)$ tenemos la parte dorsal que presenta la impresión de los escudos vertebrales en forma de Y abierta, pero sin poderle asignar posición (Figuras 3B, D).

El xifiplastron está compuesto sólo por la parte anterolateral, donde se encuentra la proyección inguinal y se une con la parte posterolateral del hipoplastron. La cara anterior de la proyección iguinal presenta varios dentículos que sirven para la unión entre placas, esta cara es más alta que ancha, el margen lateral forma casi un ángulo recto con el margen ventral y el margen medial esta inclinado, dándole una forma triangular a esta zona (Figura 3C).

Identificación. El hioplastron de Gopherus se comparó con los de Rhinoclemmys (Bataguridae) y Trachemys (Emydidae), grupos que presentan una morfología similar para esta placa, a diferencia del resto de las familias de tortugas en México. Los tres géneros presentan hioplastron con una forma general cuadrangular y un escote axilar con una prolongación axilar elevada. La diferencia de Gopherus con los géneros antes mencionados se encuentra en el escote axilar y en las impresiones de los escudos plastrales (Figura 4).

Trachemys presenta el escote axilar en forma de V, el cual se extiende posteriormente hasta la mitad del hioplastron. Las impresiones se encuentran de manera horizontal y son paralelas entre si, curvándose en la parte central de la placa (Figura 4A).

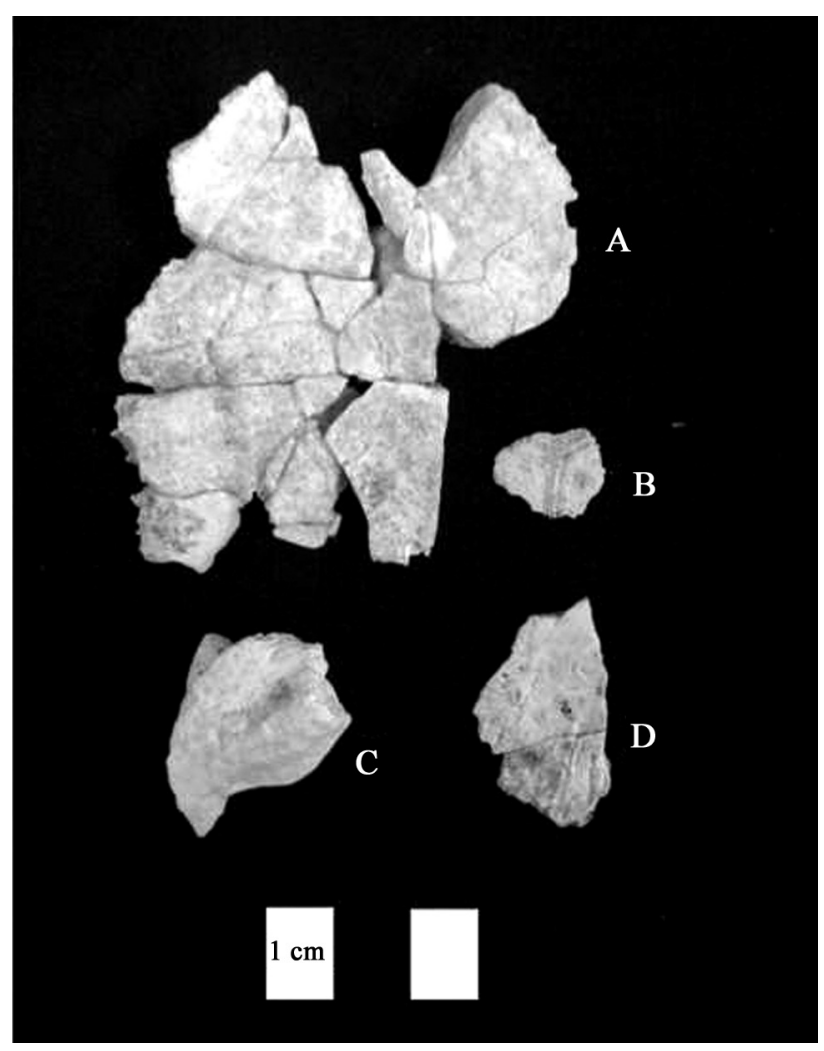

Figura 3. Material pleistocénico de Gopherus. A, vista ventral del hioplastron izquierdo (CH 14); B, vista externa de costal (CH 11); C, vista dorsal del xifiplastron derecho $(\mathrm{CH} 2)$; $\mathrm{D}$, vista interna de la primer placa costal derecha.

Rhinoclemmys presenta un escote axilar en U, la impresión húmero-pectoral se encuentra en la parte anterior del hioplastron de manera horizontal sin sobrepasar la escotadura axilar. La impresión pecto-abdominal está curvada anteriormente, disminuyendo la distancia entre impresiones en la parte central del hioplastron y aumentándola hacia la parte medial y lateral del mismo (Figura 4C).

Rhinoclemmys presenta la escotadura axilar en forma de U; al no proyectarse esta escotadura más allá de $1 / 3$ del hioplastron se puede separar de Trachemys y Gopherus. La impresión húmero-pectoral se encuentra inclinada en Gopherus, pero en Rhinoclemmys es horizontal y en Trachemys está ondulada; la impresión pecto-abdominal es recta en Gopherus y ondulada en Rhinoclemmys y Trachemys. Todo ello permite separar a Gopherus de los otros dos géneros.

El xifiplastron de Gopherus se separa del de Trachemys y Rhinoclemmys porque la proyección inguinal es más alta que ancha, ya que en el primer caso el xifiplastron es completamente plano y no presenta la proyección inguinal. En el caso de Rhinoclemmys, si se presenta dicha proyección, pero en vista anterior es más ancha que alta, el margen lateral se encuentra inclinado y el margen medial es el que presenta el ángulo recto, dando la apariencia de 
un triángulo, pero en sentido contrario que en Gopherus (Figuras 4D, E, F).

Las placas costales se asignan a Gopherus por presentar la impresión de los escudos neurales en forma de Y abierta, la ausencia de la espina costal que une esta placa con las marginales y la ausencia de marcas de crecimiento en la cara externa. Sin embargo, cabe mencionar que la presencia fragmentada de dichas placas no permite la identificación a nivel específico.

Kinosternidae Baur, 1893

Kinosternon Spix, 1824

\section{Kinosternon aff. K. hirtipes Wagler, 1830 / K. integrum LeConte, 1854}

Material recuperado. Un hioplastron izquierdo, $\mathrm{CH} 9$; una quinta placa costal derecha, $\mathrm{CH} 5$; una séptima placa costal izquierda, $\mathrm{CH} 10$; una séptima y una octava placas periféricas izquierdas, $\mathrm{CH} \mathrm{4,} \mathrm{CH}$ 6; una novena placa periférica derecha, $\mathrm{CH} 1$ (Figura 5).

Descripción. El hioplastron presenta un ancho de $39 \mathrm{~mm}$ y una longitud máxima de $19 \mathrm{~mm}$, con una forma general rectangular en posición horizontal. La expansión axilar es plana, en forma triangular y dirigida dorslamente, el margen anterior presenta tres espinas para la unión con el epiplastron (Figura 5F); en vista anterior se observa el borde ventral, el cual es delgado, encontrándose en la parte central y ocupando dos tercios de la anchura total.

Las placas periféricas fueron asignadas a su posición por las impresiones de los escudos marginales y por el borde interno, de las placas recuperadas sólo dos están completas (CH 1, CH 4).(Figuras 5A, B, C).

La posición de las placas costales fue asignada por las impresiones de los escudos vertebrales y pleurales presentes. La quinta placa costal presenta un ancho de $46.1 \mathrm{~mm}$ y una longitud de $14.2 \mathrm{~mm}$, presenta tres líneas de crecimiento en la parte ventral y las impresiones del segundo y tercer escudo vertebral y la segunda pleural. La séptima placa costal está representada por la parte dorsal con la impresión del cuarto escudo vertebral y pleural, con una longitud de $10.7 \mathrm{~mm}$ (Figuras 5D, E).

Identificación. El hioplastron de Kinosternon se diferencia por no presentar el margen que se une con el entoplastron, ya que en este género no presenta esta placa plastral, la cual está presente en el resto de los géneros con los que se compara a continuación. Además, otras características que nos confirman la identificación del material son que el hioplastron de Kinosternon se diferencia de Rhinoclemmys, Trachemys y Gopherus por presentar una forma general rectangular, más ancha que larga, la proyección axial plana y triangular. Se puede separar de Claudius (Staurotypinae) por presentar diferenciados el hio- y el hipoplastron, de Staurotypus (Staurotypinae) se diferencia porque el hioplastron presenta un escote axilar corto y el margen anterior es recto, sin curvearse anteriormente de manera

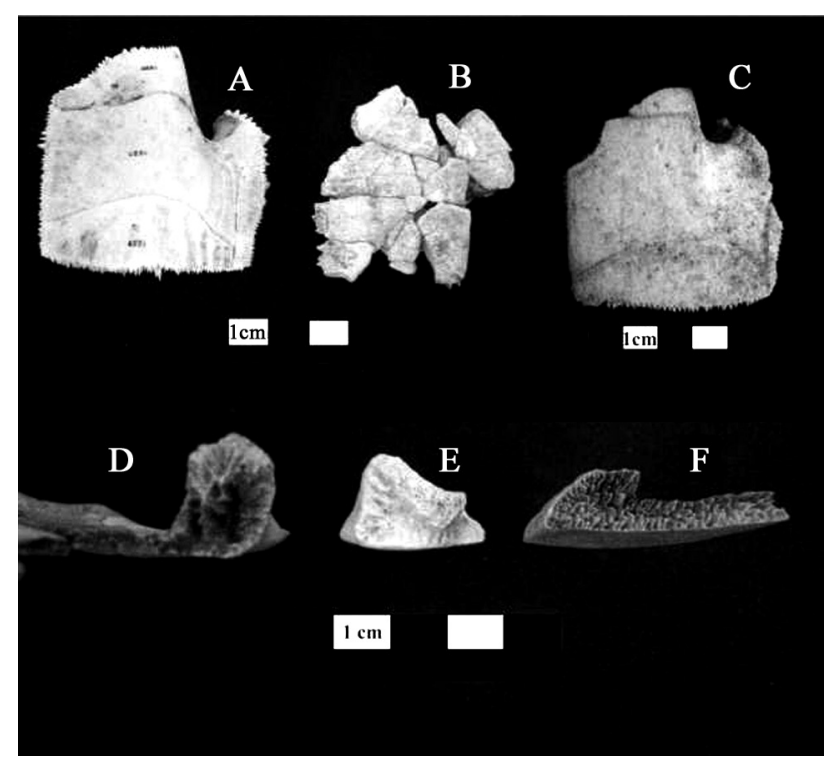

Figura 4. A, hioplastron izquierdo de Gopherus; B, hioplastron izquierdo fósil ( $\mathrm{CH}$ 14); C, hioplastron izquierdo de Rhinoclemmys; $\mathrm{D}$, xifiplastron izquierdo de Gopherus; E, xifiplastron derecho fósil (CH 2); F, xifiplastron derecho de Rhinoclemmys. A, B, C, en vista ventral; D, E, F, en vista anterior.

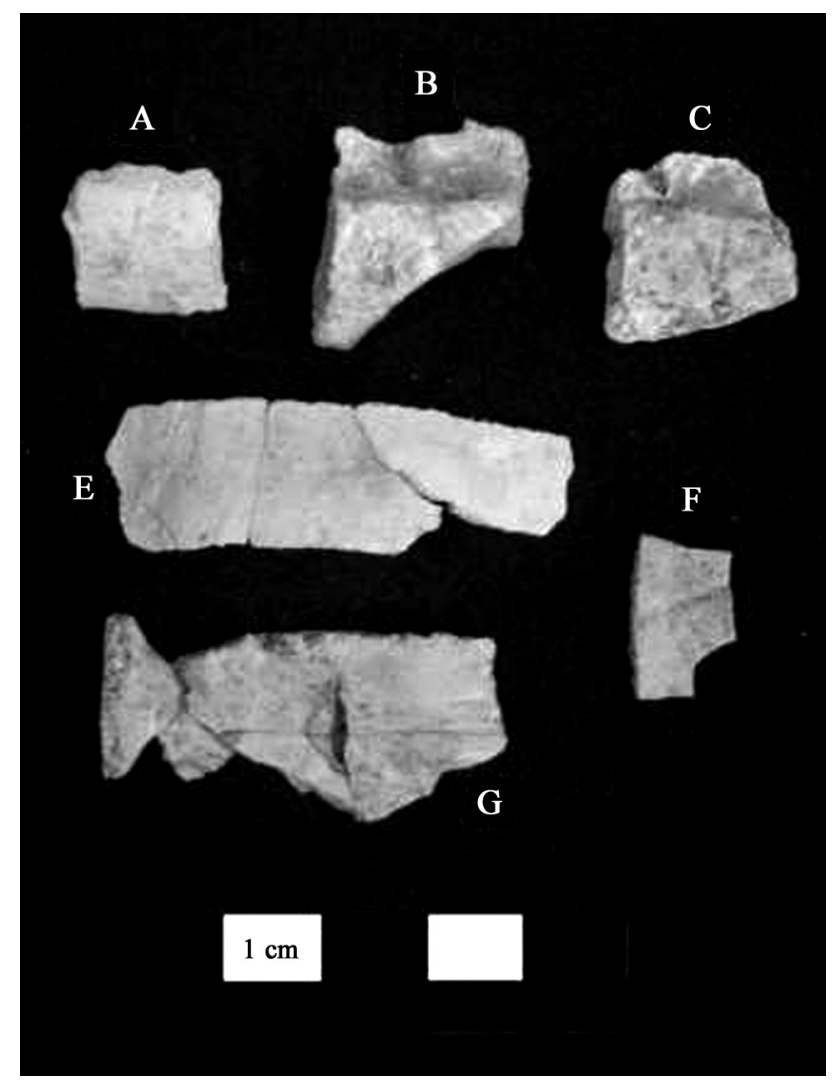

Figura 5. Material pleistocénico de Kinosternon cf. K. hirtipes o K. integrum. A, novena placa periférica derecha $(\mathrm{CH} 1) ; \mathrm{B}$, octava placa periférica izquierda (CH 6); C, séptima placa marginal izquierda (CH 4); $\mathrm{D}$, quinta placa costal derecha (CH 5); F, séptima placa costal izquierda (CH 10); G, hioplastron izquierdo (CH 9). A, B, C, G, vista interna; E, F, vista externa. 
cóncava, ambos géneros emparentados con Kinosternon. Todos los géneros antes mencionados se encuentran en el estado de Oaxaca actualmente, excepto Gopherus (CasasAndreu et al., 1996).

Las placas costales se diferencian porque la proyección del capítulo, que se une al proceso transverso de la vértebra se origina en la cara interna, bajo el margen dorsal que se une con las placas neurales, mientras que en Rhinoclemmys se origina sobre dicho margen; de Trachemys se pueden distinguir las placas costales por presentar líneas de crecimiento en la parte ventral y no sobre toda la placa; de Gopherus podemos separar estas placas por la presencia de la proyección ventral que se une a las placas periféricas, no presentes en Gopherus.

Las placas marginales se diferencian de Rhinoclemmys por presentar el foramen periferal de maneral lateral y no sobre el margen dorsal (en las placas 7 a 11); de Trachemys por la ausencia de líneas de crecimiento en la cara externa, además de presentar las caras de manera robusta y redondeadas, mientras que en Trachemys todas las caras de la placas marginales son delgadas y afiladas; por último, de Gopherus se diferencian las placas marginales por la presencia del foramen periferal.

La identificación a nivel específico se realizó comparando el material con esqueletos de cinco especies de Kinosternon actuales disponibles, las cuales son: $K$. flavescens, $K$. hirtipes, K. integrum, K. leucostomum y K. scorpioides. El hioplastron se separó porque la proyección axilar presenta una base gruesa y el ancho de esta prolongación es mayor que en $K$. flavescens, esta especie no presenta el borde ventral y la base de la proyección axilar es más delgada. El resto de las especies presentan el borde ventral, pero en el caso de $K$. leucostomum se presenta el borde muy robusto, ocupando gran parte de la altura de la placa, diferente a lo observado en el material fósil (Figura 6). El hioplastron no ayuda en la separación entre $K$. hirtipes, $K$. integrum y $K$. scorpioides.

La quinta placa costal presenta la impresión del segundo escudo vertebral en forma convexa, similar a lo hallado tanto en $K$. hirtipes y $K$. integrum, mientras que en $K$. scorpioides se presenta de manera cóncava (Figura 7A). Esta característica acerca los materiales colectados en Oaxaca a las especies $K$. integrum y $K$. hirtipes.

A pesar de que ciertas características permitieron inferir que el material fósil perteneciente a Kinosternon está relacionado con $K$. hirtipes y $K$. integrum, hay que tomar en cuenta que no se revisaron las 18 especies de Kinosternon que existen actualmente (Bickman et al., 2007). En consecuencia la determinación del material aquí reportado es tentativa, así como sugerencia a Kinosternon aff. K. hirtipes/K. integrum, especies de las cuales no se distingue el material fósil.

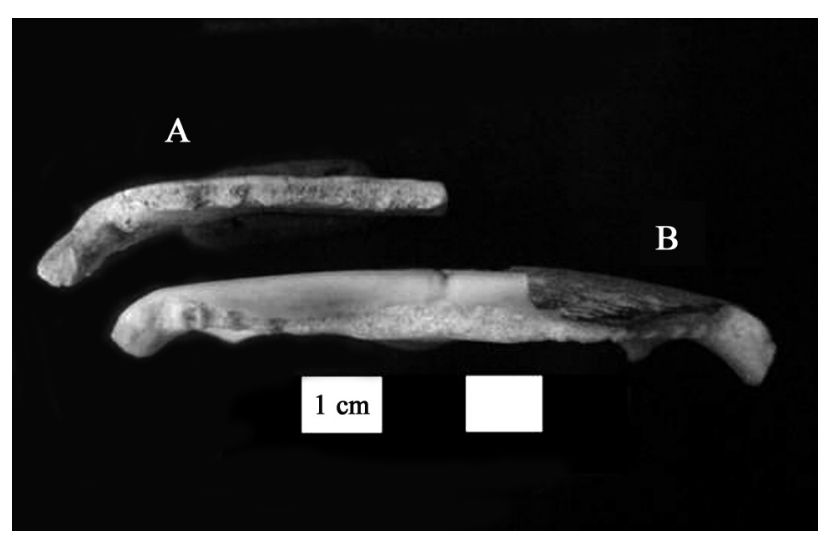

Figura 6. Comparación entre hioplastrones. A, hioplastron izquierdo fósil (CH 9); B, hioplastron izquierdo de Kinosternon leucostomum. A, $\mathrm{B}$, vista anterior.

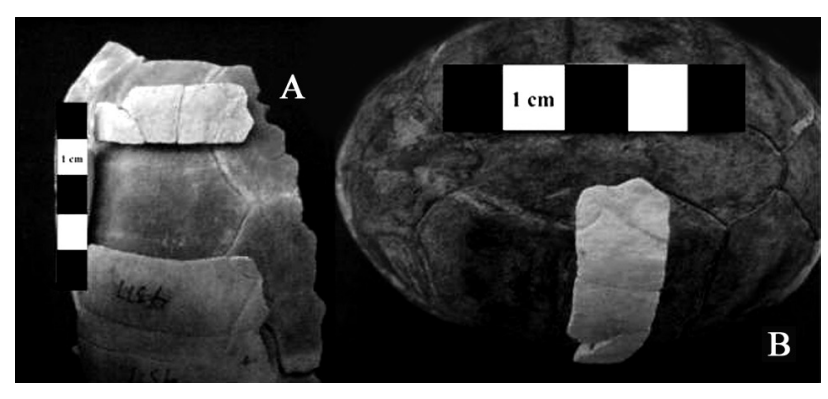

Figura 7. Comparación entre placas costales. A, fondo: fragmento de caparazón de Kinosternon integrum, frente superior: placa costal fósil (CH 5), frente inferior: fragmento de caparazón de Kinosternon hirtipes; B, fondo: caparazón de Kinosternon scorpioides, frente: placa costal fósil (CH 5).

\section{Discusión}

\subsection{Distribución.}

El género Gopherus esta constituido por un grupo de tortugas terrestres endémico de Norte América, comprende cuatro especies actualmente conocidas G. agassizii, G. berlandieri, G. flavomarginatus y G. polyphemus (Bickham et al., 2007), su distribución va desde el sur de Nevada (E.U.A.) hasta el sureste de San Luis Potosí (México). Gopherus tiene representantes fósiles desde el Oligoceno hasta el reciente, con diez especies descritas, aparte de las cuatro actuales, teniendo una distribución más amplia en el pasado (Reynoso y Montellano, 2004). En nuestro país los fósiles conocidos pertenecen al Pleistoceno y el Reciente, los registros pleistocénicos se han hallado en el norte-centro del país, Tamaulipas (Reynoso y Montellano, 2004) y Aguascalientes (Mooser, 1972, 1980), hasta el sur de Puebla (Flannery, 1967; Tovar y Montellano, 2006), en el Valle de Tehuacán-Cuicatlán, pasando por el estado de Hidalgo (Bravo-Cuevas, 2001). Por lo que los materiales identificados en este estudio representan el primer registro 
de Gopherus para el estado de Oaxaca y el tercero para el Valle de Tehuacán-Cuicatlán.

El genero Kinosternon tiene una distribución amplia en el país y para el estado de Oaxaca contamos con las especies $K$. acutum, K. integrum, K. oaxacae y K. scorpioides (Casas-Andreu et al., 1996). De los materiales identificados como Kinosternon, para K. integrum la distribución abarca desde el sur de Sonora, suroeste de Chihuahua, centro de Durango y sur de Nuevo León, hasta el centro de Oaxaca, encontrándose actualmente en el Valle de Tehuacán-Cuicatlán (Canseco-Márquez y Gutiérrez-Mayén, 2006), mostrando que esta especie se encuentra desde el Pleistoceno en el estado. K. integrum sólo ha sido reportada por Mooser (1980) para el Pleistoceno de Aguascalientes. En el caso de K. hirtipes, se mostraría una distribución más sureña durante el Pleistoceno, ya que esta se distribuye actualmente desde la región Big Bend de Texas (E.U.A.) hasta el Valle de México (Fritz y Havas, 2007) y ha sido reportada para el Pleistoceno de México en Aguascalientes (Mooser, 1980).

Durante el Pleistoceno de México la distribución de algunas especies de tortugas, cocodrilos, lagartijas y salamandras presentaron importantes cambios corológicos (e. g. García-Pérez, 2004; Reynoso y Montellano, 2004; Tovar y Montellano, 2006), muy probablemente relacionados a modificaciones climáticas y fisiográficas, así como en los microambientes y nichos ecológicos, en localidades particulares que propició la extinción de los organismos o la ocupación de otros sitios (Martínez-Meyer et al., 2004; Peterson et al., 2004).

\subsection{Escenario ambiental}

El municipio de Chazumba presenta una vegetación de tipo matorral xerófilo. Este tipo de vegetación en México cubre la mayor parte del territorio de la península de Baja California, así como grandes extensiones de la Planicie Costera y de montañas bajas de Sonora; es muy característico de amplias áreas de la Altiplanicie, desde Chihuahua y Coahuila hasta Jalisco, Guanajuato, Hidalgo y el Estado de México, prolongándose al sur en forma de faja estrecha a través de Puebla y Oaxaca. La temperatura media anual de este tipo de vegetación varia de $12-26^{\circ} \mathrm{C}$ y la precipitación media anual es en general inferior a $700 \mathrm{~mm}$ y en amplias extensiones está comprendida entre $100 \mathrm{y}$ 400mm (Rzedowski, 2006).

El matorral xerófilo es el tipo de vegetación en el que habitan Gopherus y Kinosternon hirtipes, la cual también puedes ser habitada por K. integrum, el solapamiento de sus distribuciones se da en los estados de Sonora, Sinaloa y Durango para el caso Gopherus-K. hirtipes; con Sonora, Sinaloa, Nuevo León, Tamaulipas y San Luis Potosí para Gopherus-K. integrum. Ambos solapamientos en las distribuciones indican condiciones ambientales similares con el valle de Tehuacán-Cuicatlán.

Lo anterior nos permite inferir que el ambiente en la zona de estudio fue semiárido, similar a lo encontrado por Tovar y Montellano (2006), en Santa Cruz Nuevo, Puebla, un sitio muy cercano a Chazumba, Oaxaca. Estas autoras mencionan que este tipo de ambiente existió desde el Wisconsiniano, 70,000-9,500 años AP; sin embargo, estudios palinológicos recientes para el Cuaternario tardío del valle de Tehuacán-Cuicatlan (Canul-Montañez, 2008) sugieren que el ambiente durante el Pleistoceno fue de un bosque de Pinus en el periodo comprendido entre los 25 300-12,000 $\mathrm{C}^{14} \mathrm{AP}$, y un bosque mesófilo entre los 14,000$5,000 \mathrm{C}^{14} \mathrm{AP}$, mostrando que el tipo de vegetación actual del Valle de Tehuacán-Cuicatlán es de reciente formación.

La asincronización en la formación de los ambientes áridos del norte del país con los del Valle de TehuacánCuicatlán (Canul-Montañez, 2008), sugiere el hecho de que no existan tortugas del género Gopherus en el centro de México, ya que las regiones extratropicales presentan las condiciones climáticas actuales antes que en las regiones intertropicales (Canul-Montañez, 2008), lo cual posiblemente no permitió el establecimiento de la tortuga de desierto en el Valle de Tehuacán-Cuicatlán, durante finales del Pleistoceno y principios del Holoceno, ya que este contaba con condiciones frías y húmedas. Además, el encontrar al género Gopherus en el área de estudio, puede indicar que el sitio presenta una edad mayor a los 25,000 años AP, ya que las condiciones húmedas y frías de un bosque de pino durante este periodo, no permitirían el establecimiento de dichas tortugas. Este tipo de inferencias se pueden aclarar hasta tener un fechamiento absoluto en los sitios paleontológicos estudiados en el valle, como los de Tovar y Montellano (2006) y el aquí reportado.

\section{Conclusiones}

La presencia de las tortugas Gopherus y Kinosternon aff. $K$. hirtipes o aff. $K$. integrum como fauna pleistocénica de Oaxaca es la más austral para el país para el primer género y para $K$. hirtipes, en caso de que sea confirmada la identificación, además de ser el primer registro de tortugas fósiles para el estado de Oaxaca. En el caso de Gopherus, es el tercer registro fósil para el valle de Tehuacán-Cuicatlán y el primero de Oaxaca, mientras que el registro de $K$. hirtipes es el tercero o el de $K$. integrum el segundo para el país. El haber encontrado estos taxa en la zona indica un ambiente semiárido como el que existe actualmente, indicando que este tipo de ambiente existió anterior a los 22,500 años AP, ya que los registros polínicos indican una mayor precipitación y un ambiente más frío durante el periodo comprendido entre los 22,500-5,000 años AP, para el Valle de Tehuacán-Cuicatlán.

\section{Agradecimientos}

Estamos muy agradecidos con el Municipio de Santiago 
Chazumba, Oaxaca, por la denuncia de los restos fósiles ante el INAH así como por las facilidades y el apoyo económico brindado; al proyecto de colaboración con el IPHES "Biodiversidad y sociedades cazadoras del Cuaternario de México"; a Albert Rubio y Jordi Mestre del IPHES, por la colaboración en este proyecto; a la Arq. Irán Rivera, estudiantes de la ENAH y gente del lugar (Vidal, Javier y Marcial) por participar en la excavación; al Consejo de Arqueología por la aceptación de este proyecto; al laboratorio de Arqueozoologia de la SLAA, INAH por permitirnos realizar las excavaciones en el sitio; a la Dra. María del Coro Arizmendi Arriaga por facilitar la información sobre los estudios paleoclimáticos del valle de Tehuacán-Cuicatlán; a los revisores Dr. Federico Agnolin y al Dr. Jim I. Mead por sus comentarios para enriquecer este trabajo.

\section{Referencias}

Bickham, J.W., Iverson, J.B., Parham, J.F., Philippen, H.-D., Rhodin, A.G.J., Shaffer, H.B., Spinks, P.Q., van Dijk, P.P., 2007, An Annotated list of modern turtle terminal taxa with comments on areas of taxonomic instability and recent change, in Shaffer, H.B., FitzSimmons, N.N., Georges, A., Rhodin, A.G.J. (Eds.), Defining Turtle Diversity. Proceedings of a workshop on genetics, ethics, and taxonomy of freshwater turtles and tortoises: Chelonian Research Monographs, 4,173-199.

Bravo-Cuevas, V.M., 2001, Pleistocene faunal assemblage from southcentral region of the state of Hidalgo, central Mexico: Journal of Vertebrate Paleontology, 21, 35A.

Canseco-Márquez, L., Gutiérrez-Mayén, M.G., 2006, Guía de campo de los anfibios y reptiles del Valle de Zapotitlán, Puebla: Sociedad Herpetológica Mexicana, Benemérita Universidad Autónoma de Puebla.

Canul-Montañez, M.E., 2008, Reconstrucción paleoclimática (Cuaternario Tardío) de la porción occidental del Valle de Tehuacán, Puebla, México: estudio palinológico: Instituto de Ecología, Universidad Nacional Autónoma de México, Tesis de Maestría.

Casas-Andreu, G., Méndez-de la Cruz, F.R., Camarillo, J.L., 1996 , Anfibios y reptiles de Oaxaca. Lista, distribución y conservación: Acta Zoológica Mexicana (n.s.), 69, 1-35.

Cruz Silva, J.A, 2009, Restos óseos de herpetofauna provenientes de la Cueva Encantada de Chimalacatlán, Morelos: Escuela Nacional de Ciencias Biológicas, Instituto Politécnico Nacional, Tesis de Licenciatura.

Flannery, K.V., 1967, Vertebrate fauna and hunting patterns, in Byers, D. S. (ed.), The prehistory of the Tehuacan Valley. Vol. 1: Environment and Subsistence. University of Texas Press, Austin, Texas, 132-177.
Flores-Villela, O., 1993, Herpetofauna Mexicana: Special Publications of Carnegie Museum of Natural History, 17, 1-73.

Flores-Villela, O., Canseco-Márquez, L., 2004, Nuevas especies y cambios taxonómicos para la herpetofauna de México: Acta Zoológica Mexicana (n.s.), 20(2), 115-144

Fritz, U., Havas, P., 2007, Checklist of chelonians of the world: Vertebrate Zoology, 57, 149-368.

García-Pérez, L., 2004, Caracterización de los fósiles referidos al género Crocodylus en México y un análisis de los patrones biogeográficos del género: Facultad de Ciencias, UNAM, Tesis de Licenciatura.

Liner, E.A., Casas-Andreu, G., 2008, Nombres estándar en español, en inglés y nombres científicos de los anfibios y reptiles de México. Standard spanish, english and scientific names of the amphibians and reptiles of Mexico: segunda edición, Herpetological circular No. 38, Society for de Study of Amphibians and Reptiles.

Martínez-Meyer, E., Peterson, A.T., Hargrove, W.W., 2004, Ecological niches as stable distributional constraints on mammal species, with implications for Pleistocene extinctions and climate change projections for biodiversity: Global Ecology and Biogeography, 13, 305-314.

Mooser, O., 1972, A new species of Pleistocene fossil tortoise, genus Gopherus, from Aguascalientes, Aguascalientes, México: The Southwestern Naturalist, 17, 61-65.

Mooser, O., 1980, Pleistocene fossil turtles from Aguascalientes, state of Aguascalientes: Universidad Nacional Autónoma de México, Instituto de Geología, Revista, 4,63-66.

Peterson, A.T., Martínez-Meyer, E., González-Salazar, C., 2004, Reconstructing the Pleistocene geography of the Aphelocoma jays (Corvidae): Diversity and Distributions, 10,237-246.

Reynoso, V.H., 2006, Research on fossil amphibians and reptiles in Mexico, from 1869 to early 2004 (including marine forms but excluding pterosaurs, dinosaurs, and obviously, birds), in Vega, F., Nyborg, T.G., Perrilliat, M. del C., Montellano-Ballesteros, M., CevallosFerriz, S.R.S., Quiroz-Barroso, S.A. (eds.), Studies on mexican Paleontology: Springer, Netherland, 209-231.

Reynoso, V.H., Montellano, M., 2004, A new giant turtle of the genus Gopherus (Chelonia: Testudines) from the Pleistocene of Tamaulipas, México, and a review of the phylogeny and biogeography of gopher turtles: Journal of Vertebrate Paleontology, 24, 822-837.

Rzedowski, J., 2006, Vegetación de México: 1ra Edición digital, CONABIO, México.

Tovar, R.E., Montellano, M., 2006, Pleistocene microvertebrates from Santa Cruz Nuevo, Puebla, Mexico: Current Research in the Pleistocene, 23, 72-74.

Manuscrito recibido: Febrero 27, 2009.

Manuscrito corregido recibido: Junio 19, 2009.

Manuscrito aceptado: Junio 24. 2009. 